Artículo

\title{
Transgredir las fronteras disciplinares: la transciplinariedad
}

\author{
Transgressing the disciplinary boundaries: transdisciplinarity
}

\author{
Karla Celina Rivera Hernández* \\ celina.rivera@udb.edu.sv
}

ISSN 1996-1642, Editorial Universidad Don Bosco, año 10, No.17, enero-junio de 2016, pp. 27-34

Recibido: 20 de septiembre de 2015. Aprobado: 16 de diciembre de 2015

\begin{abstract}
Resumen
La autora aborda la interdisciplinariedad a la luz del pensamiento de Edgar Morín para proponer una organización transdisciplinar de los saberes en el diseño de las propuestas curriculares, trascendiendo la organización de asignaturas inconexas y alejadas de la complejidad del mundo. Se desarrollan cuatro puntos: El primero, aborda cómo la mente rompe la complejidad del mundo en fragmentos disasociados que atrofian la posibilidad de comprender y reflexionar los fenómenos. El segundo, expone la posibilidad de reformar un modo diferente de pensamiento que integra-ordena y desordena el conocimiento. En el tercero, propone renovar el pensamiento simple por el complejo que organiza, traduce y reconstruye los conocimientos mediante separar-unir y analizar-sintetizar. Finalmente, el cuarto punto, vincula la renovación del pensamiento complejo con la reforma de la enseñanza mediante un currículo que articula los saberes para generar entendimiento interdisciplinario, asumiendo el error como un medio para el cambio y la construcción del conocimiento.
\end{abstract}

\begin{abstract}
The author approaches interdisciplinarity as an interpretation of Edgar Morin's thought to propose an interdisciplinar organization of knowledge in a curriculum that transcends the organization of subjects which are far disconnected of the complexity of the world Four ideas are developed in this paper: First, how the mind breaks complexity of the world into detached fragments that stunt the possibility to comprehend and reflect over phenomena. Second, the possibility to reform a different way of thought to integrate-orderdisorder knowledge. Third, a change of simple for complex thought to organize, translate and rebuild knowledge through divide-join, analysis-synthesis. Finally, the fourth idea, linking renewed complex thought with renewed teaching through an articulatingknowledge curriculum able to generate interdisciplinary understanding where error is assumed as a means to change knowledge construction.
\end{abstract}

Keywords: curriculum, complex thinking, interdisciplinarity, knowledge, education.

Palabras clave: Currículo, pensamiento complejo, interdisciplinariedad, conocimiento, educación.

* Maestra en Dirección de liderazgo educativo y Directora del Instituto de Investigación y Formación Pedagógica de la Universidad Don Bosco.

Para citar este artículo: Rivera, K. C. (2016). Transgredir las fronteras disciplinares: la transciplinariedad. Diálogos 17, 27-34. 
"Siempre he sentido que las verdades profundas, antagonistas las unas de las otras, eran para mí complementarias, sin dejar de ser antagonistas. Nunca he querido reducir a la fuerza la incertidumbre y la ambigüedad"

Edgar Morín

\section{Introducción}

El texto de Morín (2005) que encabeza este ensayo describe la resignificación dialéctica y creativa de los saberes reducidos y fragmentados en una nueva síntesis que los articula y contextualiza. Allí es donde aparece la complejidad como un método de construcción de saberes desde un punto de vista hermenéutico, que tiene en cuenta el entretejido de las partes, la construcción de relaciones, el caos, el cambio y la incertidumbre.

En el esfuerzo de organizar los saberes se requiere trascender las fronteras de la disciplinariedad hacia la transdisciplinariedad; es decir, percibiendo a la vez el todo y la parte, atravesando las disciplinas y trascendiendo las disciplinas especializadas con el fin de abordar los fenómenos en toda su complejidad.

El objetivo de este ensayo es desarrollar una reflexión orientada a la necesidad de renovar el pensamiento simple por un pensar complejo que traspasa las fronteras disciplinares; es decir, que logre una articulación y significación de los saberes. Se trata de tomar conciencia del error en el modo de organizar el saber en el sistema de ideas para llegar a contextualizarlo a través del pensamiento complejo que separa-une-analiza y sintetiza.

La importancia de abordar esta temática es que a través de la reflexión será posible una aproximación valorativa de las características del pensamiento simple en el currículo salvadoreño, a fin de sugerir implicaciones del pensamiento complejo, la teoría de sistemas y la transdisciplinariedad en el diseño y desarrollo de las propuestas curriculares.

\section{La fragmentación del conocimiento}

Nuestra mente siempre busca el orden, la certeza y el control. Tiende a romper con lo complejo del mundo en fragmentos disasociados y fracciona los problemas, llegando a atrofiar las posibilidades de comprensión y reflexión que impiden tomar conciencia de la diversidad de los fenómenos, en una constante organización, desorganización y reorganización.

La fragmentación del conocimiento encuentra su lógica en el conocimiento científico que durante mucho tiempo creyó haber conquistado un conjunto de verdades definitivas que reducían con exactitud las soluciones; en este sentido, describe Kunn (1989) que se llegaría a considerar como irracional el proponer

Transgredir las fronteras disciplinares: la

28. transciplinariedad el desplazamiento de una teoría por otra, en un contexto en el que lo racional era que las teorías proporcionaran nuevos ámbitos para el problema planteado: 
"Tradicionalmente se daba por supuesto que lo que cambia con un paradigma es la interpretación que hacen los científicos de las observaciones, que son fijadas de una vez por todas por la naturaleza del ambiente y del aparato perceptual".

Creímos que el conocimiento tenía un punto de partida y un término, creímos que el sol y la luna eran planetas, pero la tierra no; que los planetas giran alrededor de la tierra. Más adelante se dijo que la tierra era un planeta, el sol un astro y la luna un satélite; que los planetas giran alrededor del sol. Estas últimas afirmaciones habrían parecido graves errores de acuerdo con los conocimientos que ya eran verdades tradicionales en el contexto que surgieron.

Desde esta perspectiva, la ciencia tradicional que se caracteriza por establecer leyes que rigen el comportamiento del objeto, fundamenta la pretensión de objetividad del saber, provocando la desvinculación entre el sujeto pensante y los objetos de interés. Esa reducción en unidades conduce a la hiperespecialización que separa a las disciplinas del conocimiento debido a la falta de comunicación que se establece, provocando la ceguera o ilusión de la división del conocimiento frente a la complejidad de la realidad.

El conocimiento mutilado o dividido en parcelas, es incapaz de detectar y estudiar las realidades, llevando a abordar los fenómenos de manera parcial, siendo incapaz de resolver las problemáticas existentes en el contexto. Al respecto Morín (2005) dice que se vuelven invisibles los conjuntos complejos, las interacciones y retroacciones entre partes y todo, entidades multidimensionales, problemas esenciales.

Al fragmentar el conocimiento en parcelas, se disuelve lo esencial, haciendo imposible el aprehender, puesto que los saberes no llegan a conjugarse para constituir un pensamiento que pueda afrontar los grandes desafíos de nuestro tiempo.

\section{La realidad se impone: el cambio paradigmático}

Creímos que el conocimiento tenía un punto de partida y un término, vivimos bajo los principios de disyunción, reducción y abstracción que privan la posibilidad de conocer la ciencia misma porque no hay asociación, reflexión y engranaje entre los saberes.

Sin embargo, la realidad se impone y nos sorprende a cada instante con caos y desorden, con sus certezas e incertezas. Finalmente, se hizo evidente que los problemas particulares no pueden pensarse correctamente independientes del contexto. Es lo que Morín (2002) denomina Pensar bien: "el modo de pensar que permite aprehender en conjunto el texto y el contexto, el ser y su entorno, lo local y lo global, lo multidimensional; en resumen, lo complejo, es decir las condiciones del comportamiento humano" (p. 76).

Pensar y comprender el conocimiento como un proceso en su integralidad, orden y caos tiene como condición un cambio en nuestro modo de pensar 
en lo que ya es habitual, basado en el pensar complejo, con el fin de que podamos tener herramientas para entretejer los saberes, integrar el todo a las partes y las partes al todo. Desde este punto de vista, podemos estar ante lo que Kuhn (1989) Ilama Cambio revolucionario:

Los cambios revolucionarios ponen en juego descubrimientos que no pueden acomodarse dentro de los conceptos que eran habituales antes de que se hicieran dichos descubrimientos. Para hacer o asimilar, un descubrimiento tal, debe alterarse el modo en que se piensa y describe un rango de fenómenos naturales (p. 59).

Esto implica que el conocimiento no puede ser solamente acumulativo; sino que también es una forma diferente de interpretación del conocimiento, ordenando e integrando por si mismos los saberes de un modo nuevo y diferente que manifieste pautas que anteriormente no eran visibles.

La reforma del pensamiento pretende que todas las ciencias rompan el tradicional dogma tradicionalista de explicación por lo elemental. En este sentido es que Morín(2002) asegura que la segunda revolución científica del siglo XX puede contribuir a formar una cabeza bien puesta, en la que se llegue a vincular, contextualizar y totalizar saberes que ahora son fragmentarios $y$ compartimentados.

Tres desafíos sustentan la posibilidad de un cambio paradigmático o reforma del pensamiento; pero estos desafíos deben enfrentarse interdependientemente para establecer su vínculo:

- La fragmentación de la cultura de las humanidades y la cultura científica. La primera que enfrenta los grandes interrogantes humanos y favorece la integración personal de los conocimientos; pero sus adquisiciones científicas sobre el mundo y la vida son mantenidas en privado. Mientras que la cultura científica, provoca conocimientos y teorías geniales; pero separa los campos del conocimiento, no reflexiona sobre el destino humano y el devenir de la ciencia y priva la re flexibilidad.

- El conocimiento como capital y la información como materia prima que se deberá dominar e integrar a las actividades económicas, técnicas, sociales y políticas.

- El ciudadano pierde el derecho al conocimiento totalizador y pertinente, teniendo el derecho de adquirir el conocimiento especializado si realiza estudios ad hoc.

Transgredir las fronteras disciplinares: la

30. transciplinariedad

\section{Hacia una mente bien ordenada}

Todo lo que pasa se da en el marco de interdependencias dinámicas que producen diferentes cambios. Por ello no se pueden separar los diferentes componentes que constituyen el todo y existe un tejido interdependiente, interactivo entre las partes y el todo y el todo y las partes. 
Según Piaget (1981) el conocimiento constituye siempre un proceso de tránsito que consiste en pasar de un conocimiento menor a un estado más complejo y eficaz. Implica operaciones circulares de unión (conjunción, inclusión, implicación), separación (diferenciación, oposición, selección y exclusión) y más allá de síntesis y análisis.

Sin embargo, nuestro conocimiento desune y aísla los objetos de su contexto natural y del conjunto del que forma parte; por ello es necesario concebir qué es lo que los une, poner el conocimiento particular en su contexto y situarlo en su conjunto.

Según Piaget (1981), el conocimiento se presenta como una relación compleja entre el sujeto y los objetos. Lo importante para el conocimiento no es la serie de acciones consideradas aisladamente, sino el esquema de la generalización de las acciones mismas; es decir, "El origen de los conocimientos nunca está en la percepción sin más, sino que deriva de la totalidad de la acción cuyo esquematismo engloba la percepción superándola." (p. 81)

Dicha relación compleja entre el sujeto y los objetos posibilitarían la construcción de saberes entretejidos, que según Morín (2002) pueden ser construidos mediante el pensamiento complejo que liga los elementos entre sí estableciendo el diálogo entre las ideas para su integración en un esquema más amplio que articula los principios del orden-desorden, separación-unión, autonomía-dependencia.

En este sentido, el conocimiento escapa al campo de las disciplinas, las atraviesa desde una perspectiva transdisciplinaria; sin embargo, nuestro conocimiento va por una vía diferente caracterizada por la especialización, la fragmentación y la desunión. En la transdisciplinariedad, la cuestión clave es cómo percibir a la vez el todo y la parte, atravesando las disciplinas y trascendiendo las disciplinas especializadas, con el fin de abordar los fenómenos en toda su complejidad.

Al respecto, Morín (2002) sugiere:

La frontera disciplinaria, su lenguaje y sus conceptos propios van a aislar la disciplina en relación con las otras y en relación con los problemas que enlazan a las disciplinas. Se necesita una apertura, incluso sucede que una mirada ingenua, aficionada, fuera de la disciplina, resuelve un problema cuya solución era invisible dentro de la disciplina. La mirada ingenua, puede con frecuencia equivocadamente pero a veces con razón permitirse esta visión (p. 117).

Pensar, comprender y abordar el conocimiento en su integralidad, orden y caos tiene como condición fundamental que cambiemos nuestro modo de pensar basado en una lógica simple, por un modo de pensar complejo, para entretejer los saberes, asumir el caos y la incertidumbre como esperables y religar lo separado. En este sentido, Nicolescu (1998) distingue tres grados de interdisciplinariedad: (1) La aplicación que transfiere los métodos de una disciplina a otra conduciendo 
a nuevos descubrimientos, (2) un grado epistemológico que transfiere la lógica de una disciplina a otra generando interesantes análisis epistemológicos y (3) engendramiento de nuevas disciplinas al transferir métodos.

Finalmente, la cabeza bien puesta, nos lleva a tomar conciencia de la ilusión de que la ciencia ya había adquirido lo esencial de sus principios y resultados; sin embargo, es posible que estemos al final de un cierto tiempo y empezando tiempos nuevos.

El pensamiento que aísla y separa tiene que ser reemplazado por el pensamiento que distingue y une. Se necesita una cabeza bien puesta que esté apta para organizar, traducir y reconstruir los conocimientos pasando por el proceso circular de separar-unir-analizar y sintetizar. Morín (2002) propone 7 principios guía para un pensamiento vinculante:

1.Principio sistémico que une el conocimiento de las partes con el conocimiento del todo.

2. Principio holográmico que pone en evidencia la parte en el todo y el todo en la parte.

3. Principio del bucle retroactivo que permite el conocimiento de los procesos autorreguladores.

4. Principio del bucle recursivo en el que los productos y efectos son en sí mismos, productores y causantes de lo que se produce.

5. Principio de autonomía-dependencia que se regenera permanentemente.

6. Principio dialógico que une dos principios que deberían excluirse entre sí pero que son indisociables en una misma realidad.

7. Principio de reintroducción del que conoce en todo conocimiento, en donde todo conocimiento es una reconstrucción/traducción que hace la mente en una cultura y un tempo determinado.

\section{Aportes al proceso educativo: reforma de la enseñanza}

Según Morín (2002), la reforma del pensamiento debe conducir a la reforma de la enseñanza, y a su vez la reforma de la enseñanza conducirá a la reforma del pensamiento. Entonces en este apartado se reflexionaran los procesos formativos en el marco de las interdependencias dinámicas, de manera que sea posible la construcción e implementación de un currículo formativo que tenga en cuenta el entretejido de los saberes, la construcción de relaciones, el caos y la incertidumbre.

Uno de los rasgos que caracteriza el currículo de la educación salvadoreña es que está regido por el paradigma positivista (conductismo, pragmatismo y funcionalismo) centrado en la transmisión y acumulación acrítica del conocimientos que reproducen las pautas culturales. Sobre este esquema se diseñan currículos recargados y excesivamente fragmentados en que cada

Transgredir las fronteras disciplinares: la

32. transciplinariedad asignatura es percibida como una parcela inconexa y alejada de la complejidad del mundo; es decir un prerrequisito administrativo que no establece ninguna 
interacción en todo el recorrido formativo e inclusive en su organización interna entre las unidades de aprendizaje y contenidos agrupados por una lógica enciclopedista que atomiza el conocimiento.

Al respecto, propone Morín (2002),

Pensar el problema de la enseñanza a partir de la consideración de los efectos cada vez más graves de la compartimentación de los saberes y de la incapacidad para articularlos entre sí y, por otra parte, a partir de la consideración de que la aptitud para contextualizar e integrar es una cualidad fundamental del pensamiento humano que hay que desarrollar antes que atrofiar (p. 15).

Frente a la reforma del pensamiento, la educación reclama un nuevo paradigma acorde al contexto. Morín (2002) pretende a través de la "reforma de la educación" que se articulen los saberes para generar un entendimiento interdisciplinario y asuma el error como un medio propicio para el cambio y la construcción del conocimiento. Entonces educar no es transmitir y reproducir los conocimientos, por el contrario es una tarea que desde la interdisciplinariedad promueve la formación integral y la formulación de propuestas posibles para la solución de problemas fundamentales.

La penetración del pensamiento complejo y transdisciplinario a la educación, permitirá la evolución de la misión de las instituciones educativas. Para Nicolescu (1998) permitiría,

devenir un lugar de aprendizaje de la actitud transcultural, transreligiosa, transpolítica y transnacional, del diálogo entre el arte y la ciencia, eje de la reunificación entre la cultura científica y la cultura artística. La Universidad renovada será el hogar de un nuevo tipo de humanismo (p. 114).

\section{Conclusión}

Se producen conocimientos sin precedentes sobre el mundo bajo métodos de verificación empírica y lógica que separan, unen, jerarquizan y centralizan los saberes. Pero la visión del mundo ha cambiado y se trata de evitar una visión unidimensional que reduce y abstrae los saberes, fragmentándolos y destruyendo los conjuntos y las totalidades hasta aislar a los objetos de sus ambientes.

Se presenta para la teoría la necesidad de una lógica de unidad compleja como un todo que no se reduce a la suma de las partes y que se sitúa en un nivel transdisciplinario que asocia y organiza, que hace progresar el conocimiento frente a las alternativas clásicas, pero hace aparecer un nuevo desconocimiento. Este cambio de pensamiento, implica a su vez una reforma a la enseñanza, concretamente viabilizado a través de un currículo interdisciplinar. 


\section{Referencias}

Kuhn, T. S. (1989). La estructura de las revoluciones científicas. México: Fondo de Cultura Económica.

Morin, E. (2005). Introducción al pensamiento complejo. Barcelona: Gedisa.

Morin, E. (2002). La cabeza bien puesta. Buenos Aires: Nueva Visión.

Nicolescu, B. (1998). La transdisciplinariedad, una nueva visión del mundo. Manifiesto Centro Internacional para la Investigación Transdisciplinaria. Francia: Ediciones Du Rocher.

Piaget, J. (1981). Psicología y Epistemología. Barcelona: Ariel. 